\title{
Dependence of Single Bubble Sonoluminescence Radiation to Host Liquid, Acoustic Pressure and Noble Gas
}

\author{
Morteza Pishbini* and Ali Asghar Shokri \\ Department of Physics, Payame Noor University, PO BOX 19395-3697, Tehran, Iran; \\ morteza_pishbini@yahoo.com, aashokri@tpnu.ac.ir
}

\begin{abstract}
By using a hydrochemical simulation, the effect of host liquid, acoustic pressure and noble gas on the parameters in water for the single bubble Sonoluminescence, N-methylformamide and Sulfuric Acid with $65 \mathrm{wt} \%$, is described. The results show, with the viscosity increment of the host liquid, the SL temperature and intensity are increased. Furthermore, numerical calculation shows that, increase in the amplitude of the driving pressure from 1.3 to $1.45 \mathrm{~atm}$ and increase in the atomic mass of the noble gas from $\mathrm{Ar}$ to Xe in $\mathrm{N}$-methylformamide leads to increase in the bubble intensity by about one and two order of magnitude, respectively. The results are in excellent agreement with the experimental measurements.
\end{abstract}

Keywords: Bremsstrahlung Radiation, Hydrochemical Simulation, Single Bubble Sonoluminescence

\section{Introduction}

If the intensity of a standing sound wave are increased, this phenomena leads to pulsations and violent collapse of the bubble of gas in the liquid. This unique phenomenon named Single-bubble Sonoluminescence ${ }^{1,2}$. Since discovery of SBSL in 1989 by Gaitan ${ }^{3}$, Theoretical and Experimental researchers trying to have higher temperatures and intensities inside the bubble in the collapse time, that can lead to cold and hot fusion ${ }^{4,5}$. The results show that, the intensity of emitted light of SBSL in the collapse time is much related to different parameters, such as Ambient Temperature ${ }^{6,7}$, Ambient Pressure ${ }^{6,8}$, Noble Gas Content $^{9}$ and Host Liquid ${ }^{10,11}$. Water is a friendliest fluid for SL, but researchers report that the SL in water is about 2700 times smaller than the SL emission from sulfuric acid as a mineral acid $^{12}$. In addition, experimental reports show that SL intensity and temperature increases when in the noble gases when can see the increment of atomic mass ${ }^{13}$.

In this article, we have calculated the values of bubble properties by hydrochemical model at the time of SL emission (at maximum mode) for water, $\mathrm{N}$-methylformamide and 65 wt \% sulfuric acid as a host liquid. By thermal Bremsstrahlung Mechanism ${ }^{14}$ it is shown that, with increment of viscosity from water to acid, the SL intensity remarkably increases. In addition, we saw that increase of the amplitude of acoustic driving pressure in $\mathrm{N}$-methylformamide from 1.3 to 1.45 atm leads to increase in the SL intensity in the collapse time by about two orders of magnitude. Furthermore, in this work SL intensity and temperature from $\mathrm{N}$-methylformamide doped with different noble gases has been investigated. It is shown from Ar to Xe increased SL intensity about one order of magnitude according to the increases of the atomic mass (doped noble gas).

\section{Hydrochemical Model}

We used a hydrochemical model, which has been completely detailed in References ${ }^{15,16}$. In this model, we consider the uniform medium have bubble inside in $\mathrm{it}^{17}$. The dynamic of bubble radius is studied by the equation of Rayleigh plesset ${ }^{18}$. The physical parameters of the host liquids are shown in Table 1. In this research paper, the

${ }^{*}$ Author for correspondence 
Table 1. Physical properties of the host liquids ${ }^{20,21}$. The parameters $\mu, C, \rho, \sigma$, and $P_{\mathrm{H}_{2} \mathrm{O}}$ are Viscosity, Velocity of Sound, Density, Surface Tension and Vapor Pressure

\begin{tabular}{|c|c|c|c|c|c|c|}
\hline Host Liquid & $\boldsymbol{T}\left({ }^{\circ} \mathrm{C}\right)$ & $(\boldsymbol{\mu g} / \mathbf{m} \cdot \boldsymbol{s})$ & $\mathrm{C}(\boldsymbol{m} / \mathbf{s})$ & $\boldsymbol{\rho}\left(\mathbf{K g} / \mathbf{m}^{3}\right)$ & $\boldsymbol{\sigma}\left(\times \mathbf{1 0}^{-\mathbf{3}} \frac{\boldsymbol{N}}{\boldsymbol{M}}\right)$ & $\boldsymbol{P}_{\boldsymbol{H}_{2} \boldsymbol{O}}(\boldsymbol{P a})$ \\
\hline Water & 20 & 1.00 & 1483.0 & 998.0 & 70.70 & 2060 \\
\hline N-methylformamide & 23 & 1.65 & 1660.0 & 1000 & 38.08 & 25 \\
\hline Sulfuric Acid (65 wt \%) & 20 & 11.05 & 1565.0 & 1553.3 & 62.90 & 245 \\
\hline
\end{tabular}

formalism of boundary layer is used to describe the heat transfer between the bubble and its surrounding liquid in which the rate of exchange of thermal energy between the bubble ${ }^{19}$. The gas temperature evolution time is calculated from the equation of energy in the bubble content $^{9,19}$.

\section{Numerical Simulation and Results}

In this paper, we used the hydrochemical model for determining the parameters of SBSL in collapse time. The ultrasound frequency of the driving pressure were set at $38 \mathrm{kHz}$ for water and $65 \mathrm{wt} \%$ sulfuric acid and $36 \mathrm{kHz}$ for $\mathrm{N}$-methylformamide and the ambient pressure were 1.0 atm for all host liquids. In order to have stable SBSL that can be oscillation for a long time, three dominant instabilities including Shape ${ }^{22}$, Diffusion ${ }^{23}$ and Positional ${ }^{24}$ are considered. These instabilities can be displayed simultaneously in a zone of $\left(\mathrm{R}_{0}, \mathrm{~Pa}\right)$, which named space of phase. For water and N-methylformamide, in order to have stable bubble with maximum SL emission, the intersection of the shape instability curve with diffusion instability curve is considered. But in $65 \mathrm{wt} \%$ sulfuric acid, the point cross of the diffusion curve with the positional instability curve is the SL emission at maximum mode and this point shows the pressure of ultimate driving for oscillations (stable). This change can be due to the increment of the viscosity.

Figure 1 described the time evolutions of the bubble properties under the conditions of SL emission at maximum mode from different host liquids containing the noble gas (argon) at partial pressure (4 torr). The ambient radius axis is normalized to $\mathrm{R}_{0}$. In the time evolution of the bubble radius it is seen that, by reducing the viscosity and density of the host liquid, the number of after bounces are increased. The difference between the water and $65 \mathrm{wt} \%$ sulfuric acid SL temperature peak is about $15000 \mathrm{~K}$. In Figure 1 by using thermal bremsstrahlung mechanism of radiation the maximum SL intensity for different host liquids is indicated. The results of simulation show that increment of the fluid viscosity causes the SL intensity to increase about 40 times greater from water to $65 \mathrm{wt} \%$ sulfuric acid. In Figure 1 also the number of water vapor molecules and total number of particles inside the bubble for different host liquids is indicated. It is seen that, by decrease the water vapor pressure of the host liquid, the number of water vapor molecules inside the bubble decreased. The number of endothermic chemical reactions can be obtained from the number of water vapor molecules inside the bubble. Furthermore, from Figure 1 it is seen that at the end of collapse, the bubble in the 65 wt \% sulfuric acid has the maximum number of particles. It should be noticed that in the bubble of $\mathrm{N}$-methylformamide the number of particles doesn't remarkably change during a period and the noble gas atoms occupied the bubble.

In Figure 2 the role of acoustic driving pressure amplitude in $\mathrm{N}$-methylformamide as a host liquid has been numerically investigated. In order to have stable SBSL driving parameters are determined from the phase diagram. The maximum acoustic driving pressure amplitude is according to the time of maximum SL intensity. Our calculations show that the increment of the pressure amplitude from 1.3 to $1.45 \mathrm{~atm}$ led to SL temperature peak of the gas inside the bubble in the collapse time increased about $8000 \mathrm{~K}$.

Also we expect as shown in Figure 2 the maximum pressure amplitude has been the maximum SL intensity. It is seen that Increment of the pressure amplitude led to increase of the Ar molecules inside the bubble and as a consequence the ionized particles will be increased in the collapse time and for this reason radiation will be brighter. Results of our simulations show that the maximum SL emission in $\mathrm{P}_{\mathrm{a}}=1.3 \mathrm{~atm}$ is about 20 times smaller the SL in $\mathrm{P}_{\mathrm{a}}=1.45 \mathrm{~atm}$ in $\mathrm{N}$-methylformamide. 

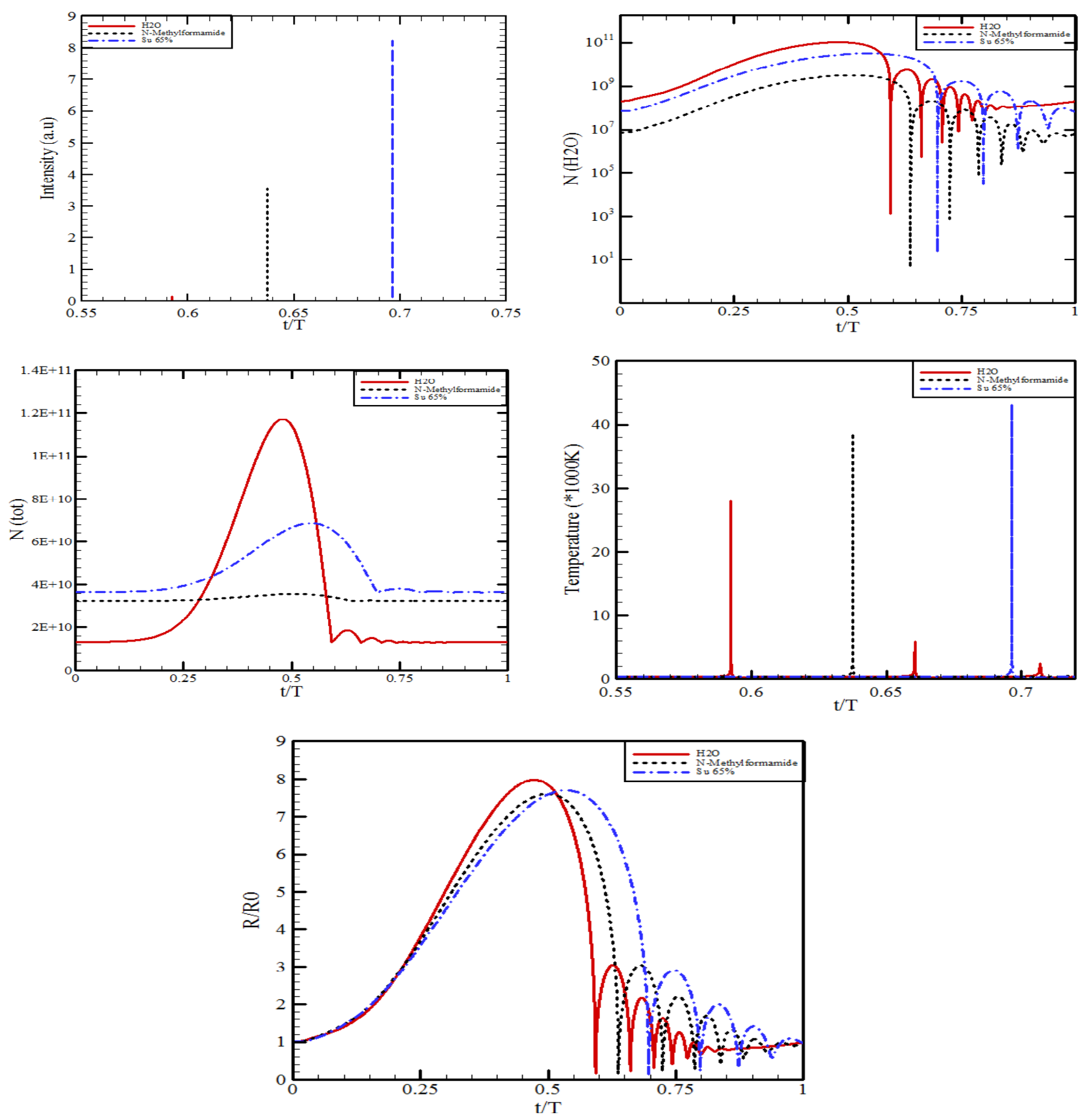

Figure 1. Time evolution of the Bubble Radius, Bubble Temperature, SL Intensity, The number of $\mathrm{H}_{2} \mathrm{O}$ particles and the total number of particles inside the bubble in three host liquid at partial pressure of 4 torr.

In Figure 3 the results of our calculations for $\mathrm{N}$-methylformamide as a host liquid and by some noble gases (Xe, $\mathrm{Kr}$ and Ar) at pressure (partial) 4 torr are presented.

The acoustic driving pressure amplitude is the same at $\mathrm{P}_{\mathrm{a}}=1.45$ atm for different noble gases in
$\mathrm{N}$-methylformamide because the change of noble gases don't be remarkably affected the shape instability curve in the phase diagram.

This pressure amplitude is corresponding by the time of maximum SL intensity. But at the collapse time and during the after bounces a remarkable difference appears. 

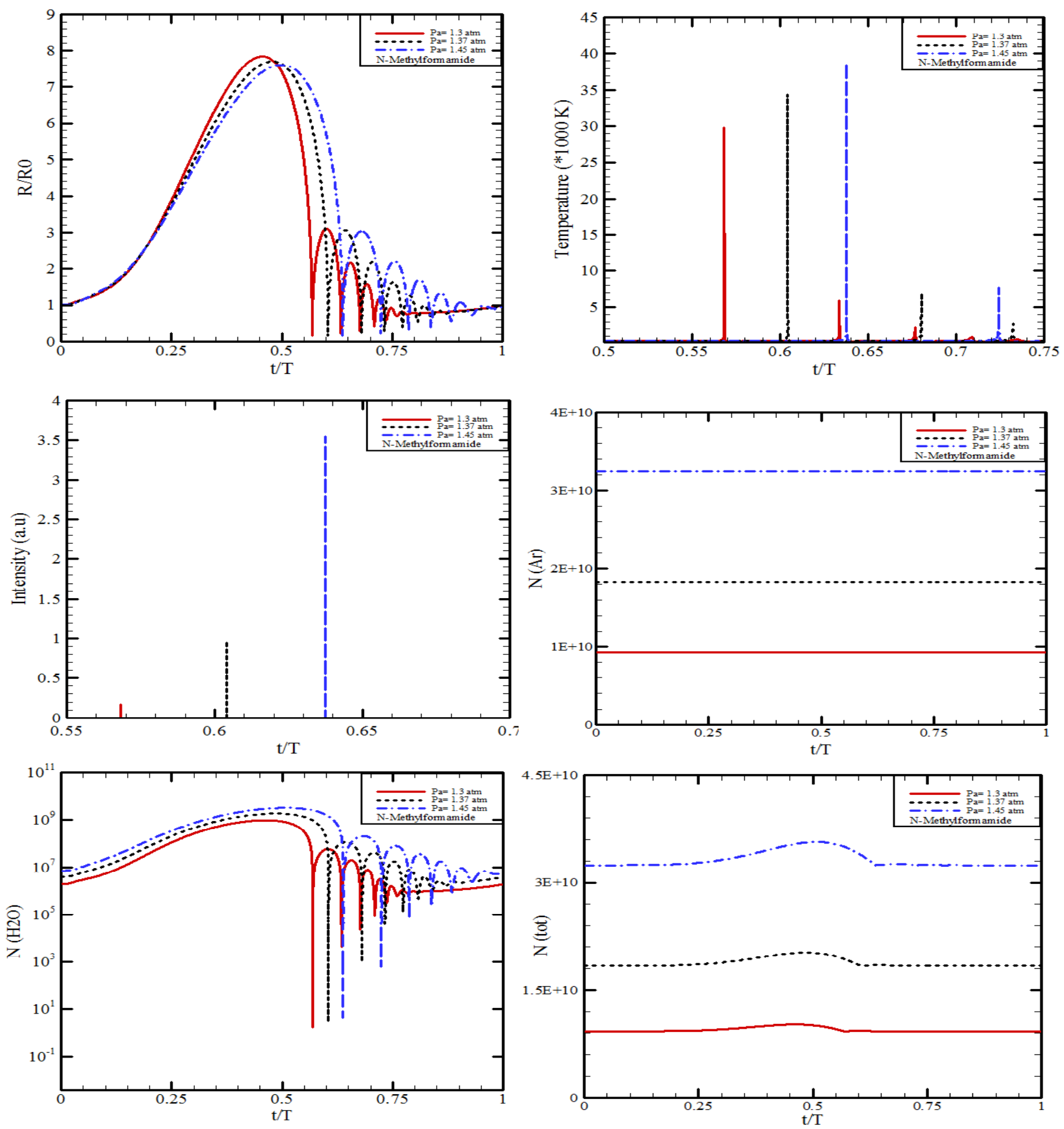

Figure 2. Time evolution of the Bubble Radius, Bubble Temperature, SL Intensity, The number of Ar particles, The number of $\mathrm{H}_{2} \mathrm{O}$ particles and the total number of particles inside the bubble in three acoustic pressure amplitude at partial pressure of 4 torr.

In addition, the Xe bubble reaches the end of collapse time before the $\mathrm{Kr}$ bubble and that also reach before $\mathrm{Ar}$ bubble. Between the three noble gases, the amplitude of after bounces for $\mathrm{Xe}$ is maximum and for $\mathrm{Ar}$ is minimum that this behavior is due to the higher atomic mass of the Xe molecules. As shown in Figure 3 heavier noble gas has a higher SL temperature and intensity. It is seen that the SL temperature and SL intensity from Xe bubble is 

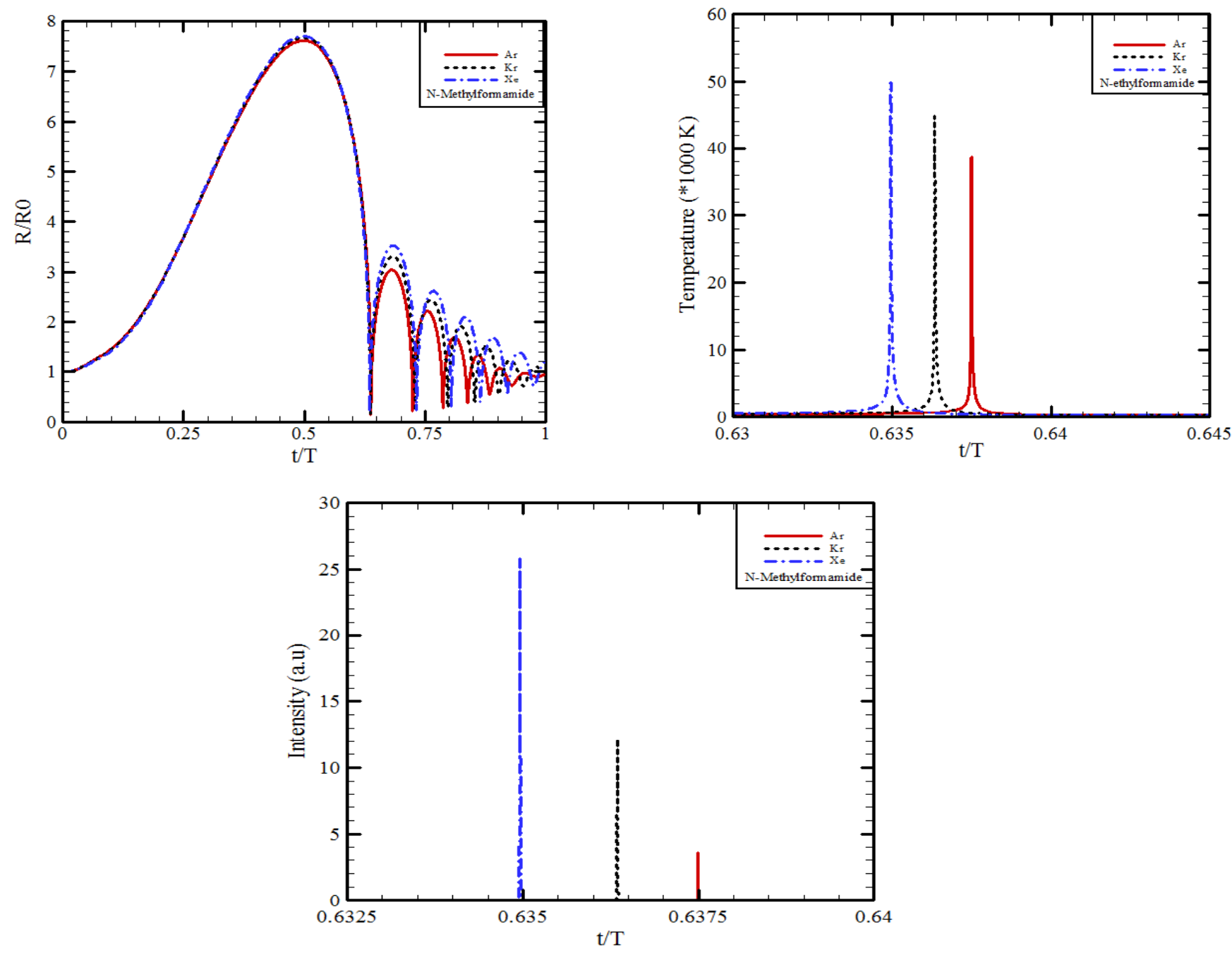

Figure 3. Time evolution of the Bubble Radius, Bubble Temperature and SL Intensity for different noble gases in $\mathrm{N}$-methylformamide at partial pressure of 4 torr.

about $10000 \mathrm{~K}$ and 8 times greater than that of Ar bubble, respectively. This increase in the temperature peak can be due to the decrease of thermal conductivity from $\mathrm{Ar}$ to Xe. Furthermore, the higher ionization energy of the Ar molecules led to decrease of the ionized particle at the collapse time and decreases the SL intensity in Ar bubble.

\section{Conclusions}

In this paper, the production of SBSL by hydrochemical model in different host liquids was studied. Furthermore, the acoustic pressure amplitude and noble gas dependency of SL in N-methylformamide was numerically investigated by calculating the maximum acquirable
SL emission. It is found that, the increment of the fluid viscosity from water to $65 \mathrm{wt} \%$ sulfuric acid led to: 1 . Time delay in the collapse time 2. Increase of the SL temperature 3. Inside the bubble (increase the number of particles) and finally 4 . Increase of the SL intensity by four orders of magnitude. The theoretically calculations also indicated that in $\mathrm{N}$-methylformamide the increment of the acoustic pressure amplitude from 1.3 to 1.45 atm led to increase of SL intensity by about two order of magnitude. In addition, simulation by different noble gases in N-methylformamide shows that SL intensity from Xe bubble is about 8 times greater than Ar bubble. In fact, this result can be due to the increment of thermal conductivity and ionization energy from Xe to Ar molecule. 


\section{References}

1. Barber BP, Putterman SJ. Observation of Synchronous Picosecond Sonoluminescence. Nature. 1991; 352(1):318-20.

2. Barber BP, et al. Defining the Unknowns of Sonoluminescence. Physics reports. 1997; 281(1):65-143.

3. Gaitan DF, et al. Sonoluminescence and Bubble Dynamics for a Single, Stable, Cavitation Bubble. J Acoust Soc Am. 1992; 91(6):3166-83.

4. Prevenslik TV. On the possibility of a cavity QED cold fusion cell. Indian J Pure and Appl Phys. 2000; 38(3):155-7.

5. Taleyarkhan RP, West CD, Cho JS, Lahey RT, Nigmatulin RI. Block RC. Additional evidence of nuclear emissions during acoustic cavitation. Phys Rev E. 2004; 69(1):0361091-11.

6. Vazquez GE, Putterman SJ. Temperature and pressure dependence of sonoluminescence. Phys Rev Lett. 2000; 85(14):3037-40.

7. Hilgenfeldt S, Lohse D, Moss WC .Water Temperature dependence of Single Bubble Sonoluminescence. Phys Rev Lett. 1998; 80(14):1332-35.

8. Dan M, Cheeke JDN, Kondic L. Dependence of Single Bubble Sonoluminescence on Ambient Pressure. Ultrasonic. 2000; 38(1):566-9.

9. Brenner MP, Hilgenfeldt S, Lohse D. Single Bubble Sonoluminescence. Rev Mod Phys. 2002; 74(2):425-84.

10. Troia A, Ripaa DM, Spagnolo R. Moving Single Bubble Sonoluminescence in Phosphoric Acid and Sulfuric Acid Solutions. Ultrason Sonochem. 2006; 13(3):278-82.

11. Moshaii A, Imani K, Silatani M. Sonoluminescence Radiation from different Concentrations of Sulfuric Acid. Phys Rev E. 2009; 80(1):0463251-7.

12. Flannigan D, Suslick SK. Plasma formation and temperature measurement during single-bubble cavitation. Nature. 2005; 434(1):52-55.
13. Xu H, Suslick K. Molecular emission and temperature measurements from single bubble sonoluminescence. Phys Rev Lett. 2010; 104(24):244301-4.

14. Yasui K. Effect of liquid temperature on sonoluminescence. Phys Rev E. 2001; 64(1):016310.

15. Lu X, Prosperetti A, Toegel R, Lohse D. Harmonic enhancement of single bubble sonoluminescence. Phys Rev E. 2003; 67(5):056310-1.

16. Toegel R, Hilgenfeldt S, Lohse D. Suppressing dissociation in sonoluminescing bubbles: The effect of excluded volume. Phys Rev Lett. 2002; 88(1):034301.

17. Moshaii A, Sadighi-Bonabi R. Role of liquid compressional viscosity in the dynamics of a sonoluminescencing bubble. Phys Rev E. 2004; 70(1):016304.

18. Keller JB, Miksis MJ. Bubble oscillations of large amplitude. J Acoust Soc Am. 1980; 68(1):628-33.

19. Imani KH, Bemani F, Silatani M, Sadighi-Bonabi R. Ambient temperature effect on single-bubble sonoluminescence in different concentrations of sulfuric acid solutions. Phys Rev E. 2012; 85(1):016329-7.

20. Gmelin L, Meyer RJ. Gmelins Handbuch Der Anorganischen Chemie (Verlag Chemie g.m.b.h). 1985.

21. Lided DR. CRC Handbook of Chemistry and Physics. CRC Press; Boca Raton; FL. 1991.

22. Toegel R, Lohse D. Phase diagrams for sonoluminescencing bubbles: A comparison between experiment and theory. J Chem Phys. 2003; 118(4):1863-75.

23. Hilgenfeldt S, Lohse D, Brenner MP. Phase diagrams for Sonoluminescing bubbles. Phys Fluids. 1996; $8(11): 2808-26$

24. Akhatov I, et al. Bjerknes force threshold for stable single bubble sonoluminescence. Phys Rev E. 1997; 55(3):3747-50. 